\title{
Iterative learning control of drop foot stimulation with array electrodes for selective muscle activation
}

\author{
M. Valtin* T. Seel* J. Raisch ${ }^{*, * *}$ T. Schauer ${ }^{*}$ \\ * Control Systems Group, Technische Universität Berlin, Berlin, \\ Germany (e-mail: valtin@control.tu-berlin.de) \\ ** Systems and Control Theory Group, Max Planck Institute for \\ Dynamics of Complex Technical Systems, Magdeburg, Germany
}

\begin{abstract}
Disorders of the central nervous system like stroke often lead to a paresis of the muscles responsible for dorsiflexion and eversion of the foot during swing phase. Functional electrical stimulation (FES) is commonly used to improve the foot movement. A precise placement of two single surface electrodes on the shank as well as a manual tuning of heelswitch triggered stimulation is required when using standard drop-foot stimulators. In this work, the use of automatically tuned array electrodes for selective nerve/muscle stimulation and the application of iterative learning control for adjusting stimulation intensities are investigated with the aim to obtain an optimal foot movement. The stimulation effect is observed by means of two inertial measurement units mounted on the foot and shank, respectively. Two array electrodes are placed over the areas covering the peroneal nerve and the muscle tibialis anterior. A fast identification procedure finds three suitable sets of array elements, forming so-called virtual electrodes, in both arrays. Two stimulation channels are established over the three virtual electrodes. Both channels produce dorsiflexion, but one promotes eversion and the other inversion. A decoupling matrix is applied to the stimulation intensities of both channels in order to obtain independent control over dorsiflexion and eversion. Finally, two independent iterative learning controllers are employed to achieve desired angle profiles. The proposed stimulation and control scheme is initially tested on a healthy person sitting on a table with the shank and foot free to swing. A tracking RMS error of less than one degree is achieved within six walking steps.
\end{abstract}

Keywords: Iterative Learning Control, Biomedical System, Array Electrodes, MIMO Decoupling, Virtual Electrodes, Functional Electrical Stimulation

\section{INTRODUCTION}

About one million strokes occur each year in the European Union (Sudlow and Warlow, 1997). Many people have walking deficits after a stroke. Ineffective dorsiflexion and eversion during the swing phase (drop foot) is present in about $20 \%$ of ambulatory stroke survivors (Wade et al., 1987). Functional electrical stimulation for correction of drop foot was introduced by Liberson et al. (1961). Stimulation systems available to patients use either a pair of surface electrodes, placed over the peroneal nerve and muscle tibialis anterior, or a two channel implanted nerve cuff electrode, to produce dorsiflexion and eversion during the swing phase of gait. The stimulation is usually triggered by a simple heel switch. Only one commercially available system involves an inclination sensor at the shank.

The electrode placement for transcutaneous stimulation represents a difficult and time consuming procedure. The stimulation response is highly sensitive, especially to the placement of the nerve stimulation electrode, and changes

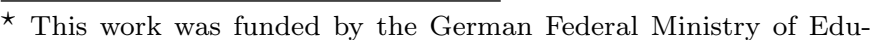
cation and Research (BMBF) within the project APeroStim (FKZ 01EZ1204B).
}

from day to day. To solve this problem, several research groups are investigating the use of array electrodes that allow a more selective nerve and muscle stimulation (Heller et al., 2012; Azevedo-Coste et al., 2007; Elsaify, 2005). Typically, all elements are individually stimulated and the corresponding foot movements are monitored by sensors mounted at the foot/shank. Elements with good responses are used to form so-called virtual electrodes inside the array electrodes.

None of the commercially available drop foot stimulators offer closed-loop control of the foot movement. The user has to adjust the stimulation intensity manually to achieve sufficient foot clearance in the swing phase. To be on the safe side, the user is tempted to choose a higher intensity than necessary. However, this leads to more rapid fatigue of the electrically stimulated muscle, which is a major limitation in functional electrical stimulation (FES).

First simple feedback control systems for dorsiflexion have been presented in (Negard, 2009; Mourselas and Granat, 2000; Hayashibe et al., 2011). While the control methods used therein yield sufficient foot clearance, they do not guarantee a natural foot motion in form of a desired dor- 
siflexion angle trajectory. That this additional objective can be achieved by the use of Iterative Learning Control (ILC) was demonstrated in (Nahrstaedt et al., 2008; Seel et al., 2013c) through simplified experiments with healthy subjects and in (Seel et al., 2013a) with stroke patients walking on a treadmill.

All mentioned closed-loop control approaches require a measurement of the foot-to-ground angle or of the dorsiflexion angle of the ankle joint, i.e. the angle between the foot and shank. The former can be determined using an inertial measurement unit $^{1}$ on the foot (Negard, 2009). In contrast, determining the joint angle requires a second IMU on the shank (Seel et al., 2013a), or alternatively a goniometer (Mourselas and Granat, 2000) or bioimpedance measurements (Nahrstaedt et al., 2008). In case inertial sensors are used, the heel switch can be replaced by a gait phase detection that uses the measured accelerations and angular rates instead (Kotiadis et al., 2010; Negard, 2009; Seel et al., 2013b; Héliot et al., 2013).

This contribution is concerned with the development of a drop foot stimulator that allows, for the first time, control of dorsiflexion and eversion during the swing phase by two decoupled iterative learning controllers. The stimulation channels are realised by automatically tuned array electrodes that cover the peroneal nerve and the muscle tibialis anterior. The proposed stimulation and control scheme are experimentally evaluated with a healthy subject.

\section{METHODS}

\subsection{Experimental Setup}

Electrode arrays with 30 and 24 elements are placed over the areas of the peroneal nerve and the muscle tibialis anterior, respectively. The flexible-PCB based arrays are custom-made. A single hydro-gel patch (AG702 Stimulating Gel, Axelgaard Manufacturing Co., Ltd., USA) with a volume resistivity of $1500 \Omega \mathrm{cm}$ is placed between electrode and skin covering all elements of an array. In this case, stimulation selectivity is ensured by a limited conductivity of the gel. The element size is $8 \times 8 \mathrm{~mm}$ and $12 \times 12 \mathrm{~mm}$ for the nerve and muscle array, respectively. The inter-element distance for both arrays is $3 \mathrm{~mm}$ in all directions. Fig. 1 shows the placement of the two array electrodes.

The slightly modified stimulation system DeltaStim (HASOMED GmbH, Germany) (Valtin et al., 2012) is used to deliver monophasic pulses between two virtual electrodes, one per array. Each virtual electrode is formed by an arbitrary subset of array elements with the help of a demultiplexer. The configuration of the virtual electrodes as well as the pulse intensities are manipulated in realtime from a PC running Linux with the Rtai-extension ${ }^{2}$. The controller design and implementation is performed in Scilab/Scicos 4.1.2 using the HART-toolbox ${ }^{3}$. This toolbox supports automatic real-time code generation based on Rtai-Lab. The GUI QRtaiLab ${ }^{4}$ is used for signal monitoring and parameter updates.

\footnotetext{
1 i.e. typically a combination of accelerometers, gyroscopes, and magnetometers

2 http://www.rtai.org

3 http://hart.sf.net

4 http://qrtailab.sf.net
}

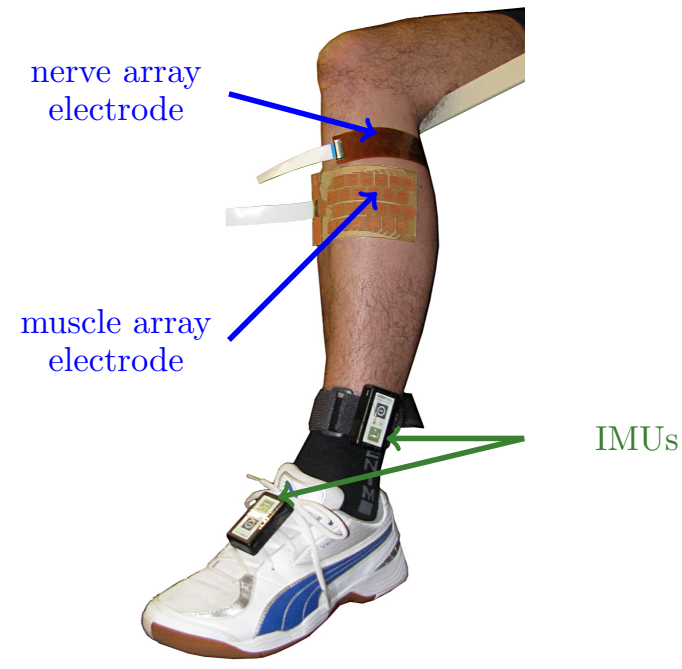

Fig. 1. Experimental setup showing the array electrodes and inertial motion units (IMUs) at the left leg.

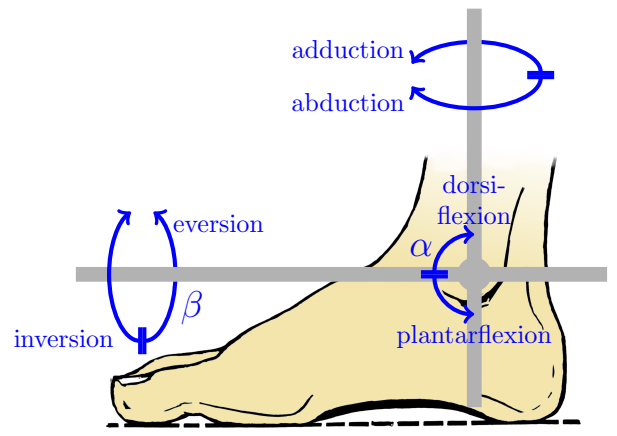

Fig. 2. Definition of fundamental movements of the foot.

When applying monophasic pulses, the majority of muscle action potentials will be generated under the array acting as cathode (active electrode), especially for a large anodic (passive) counter electrode (Reilly and Diamant, 2011). All pulses are current-controlled and the injected charge $q$ is defined as stimulation intensity. The muscle contraction is adjusted by this control signal. The charge itself is defined as product of the current amplitude $I$ and the pulsewidth $p w$. In this application, a given charge is equally distributed to pulsewidth and current amplitude (normalised to their maximal values) as follows:

$p w=\sqrt{\frac{q p w_{\max }}{I_{\max }}}, \quad I=\sqrt{\frac{q I_{\max }}{p w_{\max }}}, \quad 0 \leq q \leq\left(I_{\max } p w_{\max }\right)$, where $p w_{\max }=500 \mu \mathrm{s}$ and $I_{\max }=60 \mathrm{~mA}$ are the chosen upper limits for pulse width and current amplitude.

Two inertial measurement units (IMUs) (RehaWatch, HASOMED GmbH, Germany) are mounted on the shank and foot to determine the dorsiflexion angle $\alpha$ as well as the eversion angle $\beta$ of the foot. Data are transmitted via Bluetooth from the sensors to the PC.

Since the IMUs are attached in arbitrary orientation to the shank and foot, a calibration movement is used to identify the sensor specific rotation matrices which transform the $100 \mathrm{~Hz}$ measurement data from the sensor coordinate systems into shank- and foot-related coordinate systems. 
The rotated accelerometer, gyroscope, and magnetometer data are used to calculate each sensor's orientation using a direction cosine matrix (DCM) algorithm. The resulting Euler angles describe the sensor orientations in a common global earth coordinate system. In order to obtain the foot dorsiflexion angle $\alpha$ (cf. Fig. 2), the pitch angles from the foot and shank sensor are subtracted. The eversion angle $\beta$ is calculated by subtraction of the roll angles of both sensors.

\subsection{Identification of Virtual Electrodes}

The following procedures are carried out automatically while the subject is sitting on a table with the shank and foot free to swing:

Determine a stimulation intensity for the identification: In a first step, a suitable stimulation intensity is determined which will later be used for identifying good elements in both muscle and nerve array. The chosen intensity should yield, at least for some array elements, sufficient dorsiflexion without discomfort when stimulating exclusively over these single elements. Usually the dorsiflexion related to nerve stimulation is less than the one related to muscle stimulation. Therefore, we find this stimulation intensity for nerve stimulation using the entire muscle array as passive counter electrode while applying a sequence of monophasic stimulation pulses to all individual elements of the nerve array in a loop. Every nerve array element is stimulated with three pulses at $25 \mathrm{~Hz}$ before switching to the next element. The stimulation intensity starts at $0.05 \mu \mathrm{As}$ and is increased in steps of $0.05 \mu \mathrm{As}$ after every completed loop until the continuously measured dorsiflexion exceeds ten degrees. The so found stimulation intensity is called $q_{\text {ident }}$.

Identifying suitable array elements: In a second step, suitable elements for the muscle and nerve stimulation are selected. For each array electrode, elements with good dorsiflexion are identified by applying monophasic pulses at $25 \mathrm{~Hz}$ for $0.5 \mathrm{~s}$ with intensity $q_{\text {ident }}$ to each element while using the entire other array as counter electrode. Monophasic impulses guarantee almost pure nerve and muscle stimulation, respectively. A pause of $1.5 \mathrm{~s}$ is used between the stimulation of the individual elements to allow the foot to return to the rest position. For both the nerve and the muscle array, the four elements with the highest dorsiflexion form a virtual electrode, the virtual nerve and muscle electrode, respectively. For the nerve array electrode, a second set of electrodes, which does not show a stimulation effect, is identified and used to form a virtual passive counter electrode for the muscle stimulation. Based on these results, two time-multiplexed monophasic stimulation channels are obtained: A muscle stimulation channel with the virtual electrode on the muscle as cathode and the virtual passive counter electrode over the nerve as anode; as well as a nerve stimulation channel employing the virtual electrode on the nerve as cathode and the virtual electrode on the muscle as anode. The changing polarities guarantee a certain degree of charge balance with respect to the entire array electrodes.

Both channels produce dorsiflexion, but muscle stimulation promotes usually a small amount of inversion while nerve stimulation promotes eversion. The stimulation in-

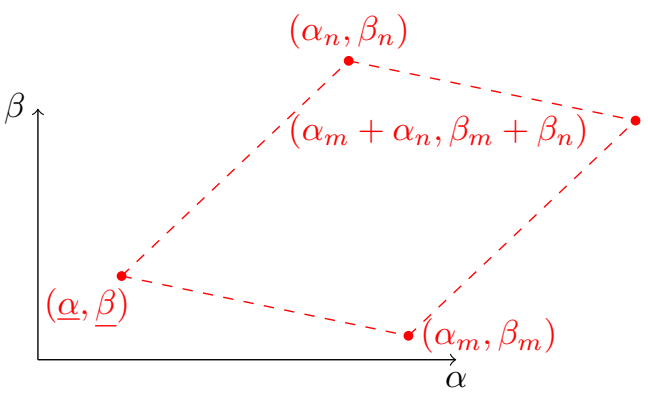

Fig. 3. Theoretically feasible FES-actuated area of dorsiflexion and inversion/eversion.

tensities of the muscle and nerve stimulation are $q_{m}$ and $q_{n}$, respectively.

\subsection{Decoupling of Stimulation Channels}

The two stimulation channels will be statically decoupled to enable independent control of dorsiflexion and eversion. Let $\underline{q}_{n}$ and $\underline{q}_{m}$ be the stimulation intensities at which the foot starts to move. The stimulation intensities $\bar{q}_{n}$ and $\bar{q}_{m}$ describe the maximal stimulation intensities that are well tolerated by the user and that yield enough functional dorsiflexion. No saturation effects in the foot angles should be visible. For the defined stimulation intensity ranges, a linear static relation between the stimulation intensities and the resulting angles is assumed:

$$
\left(\begin{array}{c}
\alpha-\underline{\alpha} \\
\beta-\underline{\beta}
\end{array}\right)=\left(\begin{array}{ll}
c_{\alpha, m} & c_{\alpha, n} \\
c_{\beta, m} & c_{\beta, n}
\end{array}\right)\left(\begin{array}{c}
q_{m}-\underline{q}_{m} \\
q_{n}-\underline{q}_{n}
\end{array}\right), \quad \underline{q}_{m} \leq q_{m} \leq \bar{q}_{m} \leq \underline{q}_{n} \leq \bar{q}_{n}
$$

Here, $\underline{\alpha}$ and $\beta$ are the minimal angles observed at minimal stimulation intensities $\underline{q}_{n}$ and $\underline{q}_{m}$. A typical feasible region for $\alpha$ and $\beta$ is depicted in Fig. 3. In order to identify the coefficients $c_{i, j}, \quad i \in\{\alpha, \beta\}, \quad j \in\{n, m\}$, the angles $\left(\alpha_{m}, \beta_{m}\right)$ and $\left(\alpha_{n}, \beta_{n}\right)$ at maximal pure muscle stimulation $\left(\bar{q}_{m}, \underline{q}_{n}\right)$ and nerve stimulation $\left(\underline{q}_{m}, \bar{q}_{n}\right)$ are measured. Choosing

$$
\begin{aligned}
\left(\begin{array}{l}
q_{m} \\
q_{n}
\end{array}\right) & =\left(\begin{array}{ll}
c_{\alpha, m} & c_{\alpha, n} \\
c_{\beta, m} & c_{\beta, n}
\end{array}\right)^{-1}\left(\begin{array}{l}
u_{\alpha}-\underline{\alpha} \\
u_{\beta}-\underline{\beta}
\end{array}\right)+\left(\begin{array}{l}
\underline{q}_{m} \\
\underline{q}_{n}
\end{array}\right) \\
& =:\left(\begin{array}{ll}
\tilde{c}_{m, \alpha} & \tilde{c}_{m, \beta} \\
\tilde{c}_{n, \alpha} & \tilde{c}_{n, \beta}
\end{array}\right)\left(\begin{array}{l}
u_{\alpha}-\underline{\alpha} \\
u_{\beta}-\underline{\beta}
\end{array}\right)+\left(\begin{array}{l}
\underline{q}_{m} \\
\underline{q}_{n}
\end{array}\right)
\end{aligned}
$$

with the new inputs $u_{\alpha}$ und $u_{\beta}$, statically decouples the system. The new inputs must lie inside the rhomboid shown in Fig. 3 to yield unconstrained stimulation intensities.

\subsection{Iterative Learning Control}

Iterative learning control is a method that aims at improving the performance of a system which executes the same task multiple times. These improvements are achieved by learning from previous trials/iterations/passes (Bristow et al., 2006). Due to the repetitive nature of gait, ILC is a promising tool for the control of drop foot stimulation. Since we decoupled the stimulation dynamics, two separate SISO ILC problems can be formulated for dorsiflexion and eversion, respectively. Due to the inherent need of data storage, discrete-time is considered the natural domain for ILC analysis (Bristow et al., 2006). 
With $\nu$ being the relative degree of the two decoupled single-input single-output systems, let

$$
\begin{aligned}
\boldsymbol{u}_{i, j} & =\left[u_{i, j}(1), u_{i, j}(2), \ldots, u_{i, j}(N)\right]^{T} \in \mathbb{R}^{N}, \\
\boldsymbol{y}_{i, j} & =\left[y_{i, j}(1+\nu), y_{i, j}(2), \ldots, y_{i, j}(N+\nu)\right]^{T} \in \mathbb{R}^{N}, \\
\boldsymbol{v}_{i} & =\left[v_{i}(1+\nu), v_{i}(2+\nu), \ldots, v_{i}(N+\nu)\right]^{T} \in \mathbb{R}^{N}
\end{aligned}
$$

be the lifted signal vectors (i.e. the vectors of $N$ sequent sample values) of the input signals, of the output signals, and of unknown, but iteration-invariant, disturbance signals for both control problems $i \in\{\alpha, \beta\}$, respectively. Furthermore, $N \in \mathbb{N}$ is the pass length and $j \in \mathbb{N}_{0}$ is the iteration/trial/pass index. The output signals of iteration $j$ are $y_{\alpha, j}(k)=\alpha_{j}(k)$ and $y_{\beta, j}(k)=\beta_{j}(k), k=1, \cdots, N$, respectively. Angle errors $\boldsymbol{e}_{i, j}=\left(\boldsymbol{y}_{i}^{d}-\boldsymbol{y}_{i, j}\right) \in \mathbb{R}^{N}$ are defined with respect to desired outputs $\boldsymbol{y}_{i}^{d} \in \mathbb{R}^{N}, i \in\{\alpha, \beta\}$, and the goal of the ILC is to reduce the error (in some norm) to a small number from pass to pass. To this end, we employ the following input update laws

$$
\boldsymbol{u}_{i, j+1}=\boldsymbol{Q}_{i}\left(\boldsymbol{u}_{i, j}+\boldsymbol{L}_{i} \boldsymbol{e}_{i, j}\right), i \in\{\alpha, \beta\},
$$

where $\boldsymbol{Q}_{i}, \boldsymbol{L}_{i} \in \mathbb{R}^{N \times N}$ are the so-called $\boldsymbol{Q}$-filters and learning gain matrices, respectively.

The $\boldsymbol{Q}_{i}$-filter matrices are designed in form of non-causal second-order low-pass filters with a cut-off frequency of $2.5 \mathrm{~Hz}$ to obtain smooth stimulation intensity profiles and to increase robustness (Bristow et al., 2006).

Furthermore, a diagonal learning matrix $\boldsymbol{L}$ is used:

$$
\boldsymbol{L}_{i}(\lambda):=\lambda_{i} \boldsymbol{I}, \lambda_{i} \in \mathbb{R}, i \in\{\alpha, \beta\} .
$$

The values of $\lambda_{\alpha}$ and $\lambda_{\beta}$ are chosen and adapted experimentally based on experience from previous work (Seel et al., 2013c,a).

When implementing the two ILCs, the feasible range for the reference signals (cf. Fig 3) and control signals, either transformed control inputs or original stimulation intensities, have to be taken into account. For the transformed control inputs, we formulate the two ILC update laws as follows:

$$
\left(\begin{array}{l}
\boldsymbol{u}_{\alpha, j+1} \\
\boldsymbol{u}_{\beta, j+1}
\end{array}\right)=\left(\begin{array}{ll}
\boldsymbol{Q}_{i} & \mathbf{0}_{N} \\
\mathbf{0}_{N} & \boldsymbol{Q}_{i}
\end{array}\right)\left[\left(\begin{array}{l}
\boldsymbol{u}_{\alpha, j} \\
\boldsymbol{u}_{\beta, j}
\end{array}\right)+\left(\begin{array}{cc}
\boldsymbol{L}_{i} & \mathbf{0}_{N} \\
\mathbf{0}_{N} & \boldsymbol{L}_{i}
\end{array}\right)\left(\begin{array}{l}
\boldsymbol{e}_{\alpha, j} \\
\boldsymbol{e}_{\beta, j}
\end{array}\right)\right],
$$

where $\mathbf{0}_{N}$ is the zero matrix of dimension $N \times N$. We express the transformed control signal vectors $\boldsymbol{u}_{\alpha, j}$ and $\boldsymbol{u}_{\beta, j}$ by the stimulation intensity vectors

$$
\begin{array}{r}
\boldsymbol{q}_{i, j}=\quad\left[q_{i, j}(1-\nu), q_{i, j}(2-\nu), \ldots, q_{i, j}(N-\nu)\right], \\
i \in n, m,
\end{array}
$$

and use

$$
\begin{aligned}
\left(\begin{array}{c}
\boldsymbol{u}_{\alpha, j} \\
\boldsymbol{u}_{\beta, j}
\end{array}\right)= & \underbrace{\left(\begin{array}{ccc}
\tilde{c}_{m, \alpha} \boldsymbol{I}_{N} & \tilde{c}_{m, \beta} \boldsymbol{I}_{N} \\
\tilde{c}_{n, \alpha} \boldsymbol{I}_{N} & \tilde{c}_{n, \beta} \boldsymbol{I}_{N}
\end{array}\right)}_{\tilde{\boldsymbol{C}}}-\left(\begin{array}{c}
\boldsymbol{q}_{m, j}-\underline{q}_{m} \mathbf{1}_{N \times 1} \\
\boldsymbol{q}_{n, j}-\underline{q}_{n} \mathbf{1}_{N \times 1}
\end{array}\right) \\
& +\left(\begin{array}{c}
\hat{\beta} \mathbf{1}_{N \times 1} \\
\underline{\alpha} \mathbf{1}_{N \times 1}
\end{array}\right),
\end{aligned}
$$

where $\mathbf{1}_{N \times 1}$ is a ones vector of dimension $N \times 1$ and $\boldsymbol{I}_{N}$ is the identity matrix of dimension $N \times N$. With this, we rewrite the above ILC input update law for stimulation intensities. Furthermore, we introduce antiwindup to account for saturation of stimulation intensities. Thereby, the following modified ILC input update law is obtained:

$$
\begin{aligned}
& \left(\begin{array}{c}
\boldsymbol{q}_{m, j+1}^{*} \\
\boldsymbol{q}_{n, j+1}^{*}
\end{array}\right)=\tilde{\boldsymbol{C}}\left(\begin{array}{cc}
\boldsymbol{Q}_{i} & \mathbf{0}_{N} \\
\mathbf{0}_{N} & \boldsymbol{Q}_{i}
\end{array}\right)\left[\tilde{\boldsymbol{C}}^{-1}\left(\begin{array}{c}
\boldsymbol{q}_{m, j}-\underline{q}_{m} \mathbf{1}_{N \times 1} \\
\boldsymbol{q}_{n, j}-\underline{q}_{n} \mathbf{1}_{N \times 1}
\end{array}\right)\right. \\
& \left.+\left(\begin{array}{c}
\underline{\beta} \mathbf{1}_{N \times 1} \\
\underline{\alpha} \mathbf{1}_{N \times 1}
\end{array}\right)+\left(\begin{array}{cc}
\boldsymbol{L}_{i} & \mathbf{0}_{N} \\
\mathbf{0}_{N} & \boldsymbol{L}_{i}
\end{array}\right)\left(\begin{array}{l}
\boldsymbol{e}_{\alpha, j} \\
\boldsymbol{e}_{\beta, j}
\end{array}\right)\right] \\
& -\tilde{\boldsymbol{C}}\left(\frac{\beta \mathbf{1}_{N \times 1}}{\underline{\alpha} \mathbf{1}_{N \times 1}}\right)+\left(\begin{array}{l}
\underline{q}_{m} \mathbf{1}_{N \times 1} \\
\underline{q}_{n} \mathbf{1}_{N \times 1}
\end{array}\right)
\end{aligned}
$$

with

$$
\begin{aligned}
& q_{i, j+1}(k)=\operatorname{sat}\left(q_{i, j+1}^{*}(k)\right) \\
& = \begin{cases}\bar{q}_{i} & , q_{i, j+1}^{*}(k)>\bar{q}_{i} \\
q_{i, j+1}^{*}(k) & , \underline{q}_{i} \leq q_{i, j+1}^{*}(k) \leq \bar{q}_{i} \\
\underline{q}_{i} & , q_{i, j+1}^{*}(k)<\underline{q}_{i}\end{cases} \\
& \forall k=1, \ldots, N, \quad i \in n, m \text {. }
\end{aligned}
$$

\section{RESULTS}

In a series of experiments, which were approved by the Ethics Committee at Charité Berlin, we evaluated the proposed stimulation and control system with a healthy subject. The person was sitting on a table with the shank and foot free to swing.

Figs. 4 and 5 illustrate the results for a virtual electrode identification, which took 108 seconds. The observed maximal changes in dorsiflexion/plantarflexion and eversion/inversion with respect to the rest position of the hanging foot are plotted for each individually stimulated element as a vector. The length of the vector represents the amount of dorsiflexion/plantarflexion. If a vector points upwards then dorsiflexion occurred, otherwise plantarflexion was observed. Vectors pointing to the left indicate inversion and vectors to the right indicate eversion. The chosen virtual electrodes are indicated as well.

Fig. 6 shows exemplary stimulation responses for the orginal, non-decoupled system. System inputs and outputs for muscle stimulation are reported in the left graphs. The results for nerve stimulation are shown in the right subplots. The coupling of the stimulation channels is clearly visible. The Relative Gain Array (RGA) (Bristol, 1966) is given by

$$
R G A_{\text {original }}=\left(\begin{array}{ll}
\rho_{\alpha, q_{m}} & \rho_{\alpha, q_{n}} \\
\rho_{\beta, q_{m}} & \rho_{\beta, q_{n}}
\end{array}\right)=\left(\begin{array}{cc}
0.777 & 0.223 \\
0.223 & 0.777
\end{array}\right) .
$$

In Fig. 7, input step responses of the decoupled system with the new inputs $u_{\alpha}$ and $u_{\beta}$ are presented. The corresponding RGA matrix is

$$
R G A_{\text {new }}=\left(\begin{array}{ll}
\rho_{\alpha, u_{\alpha}} & \rho_{\alpha, u_{\beta}} \\
\rho_{\beta, u_{\alpha}} & \rho_{\beta, u_{\beta}}
\end{array}\right)=\left(\begin{array}{cc}
1.03 & -0.03 \\
-0.03 & 1.03
\end{array}\right) .
$$

The expected decoupling effect can be clearly observed.

The iterative learning control of dorsiflexion and eversion was tested for simulated swing phases with a duration of $1.2 \mathrm{~s}$. A pause of two seconds was inserted between ILC iterations (swing phases) to allow the foot to return to the rest position and to delay fatigue.

The learning gains $\lambda_{\alpha}$ and $\lambda_{\beta}$ have been set to 0.5 and 0.2 , respectively. This value has been chosen based on previous results for pure dorsiflexion ILC (Seel et al., 2013c).

Figs. 8 and 9 illustrate the performance of the ILCs. The reference profiles for both angles guide the foot smoothly from the rest position to desired positive values 


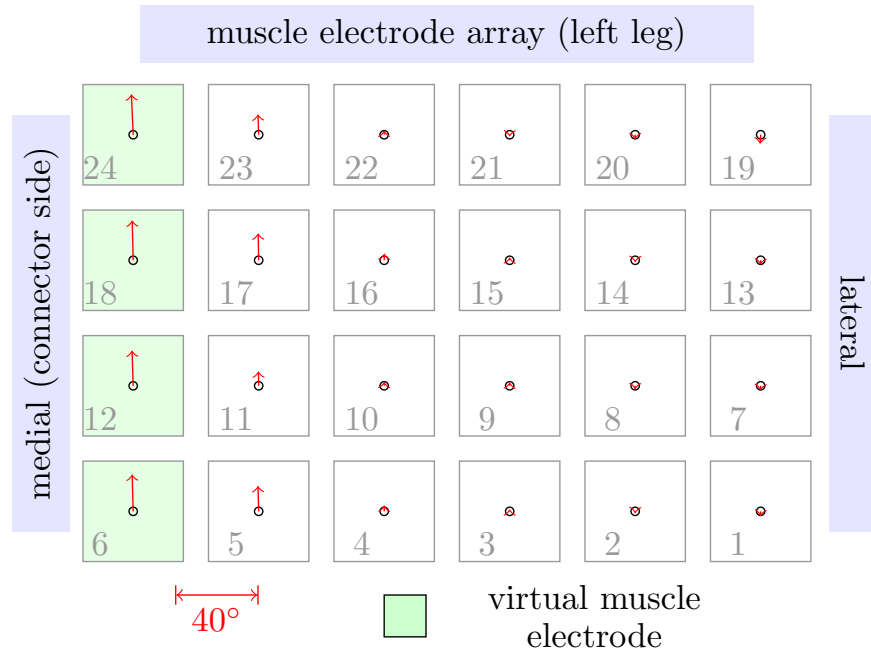

dorsiflexion $(\alpha>0)$

inversion $(\beta<0) \longleftrightarrow$ eversion $(\beta>0)$
plantarflexion $(\alpha<0)$

Fig. 4. Result of virtual electrode identification in the muscle array electrode.

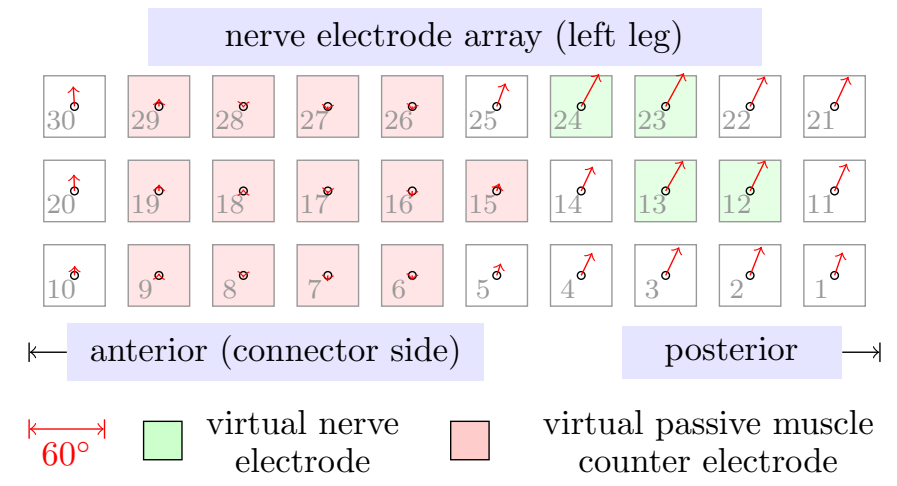

Fig. 5. Result of virtual electrodes identification in the nerve array electrode.

of dorsiflexion and eversion. The stimulation starts $200 \mathrm{~ms}$ before the actual foot movement due to the approximate relative degree $\nu=5$ at a stimulation sample period of $40 \mathrm{~ms}$. It should be noted, that the stimulation intensity $q_{m}$ for the muscle stimulation temporary saturated at $6 \mu \mathrm{As}$ in the shown trials three and five. Despite this fact, convergence to a non-saturated control signal profile was achieved. The RMS error for both angle profiles decreased below one degree within six iterations.

\section{CONCLUSIONS}

We proposed the first closed-loop control system for drop foot stimulation that allows simultaneous regulation of dorsiflexion and eversion. Virtual stimulation electrodes within two array electrodes have been automatically determined by monitoring the stimulation effect with inertial measurement units at the shank and foot. A physiological foot movement in the swing phase was achieved by means of two decoupled ILCs in a few "walking" steps. A simple linear static model was assumed for designing the
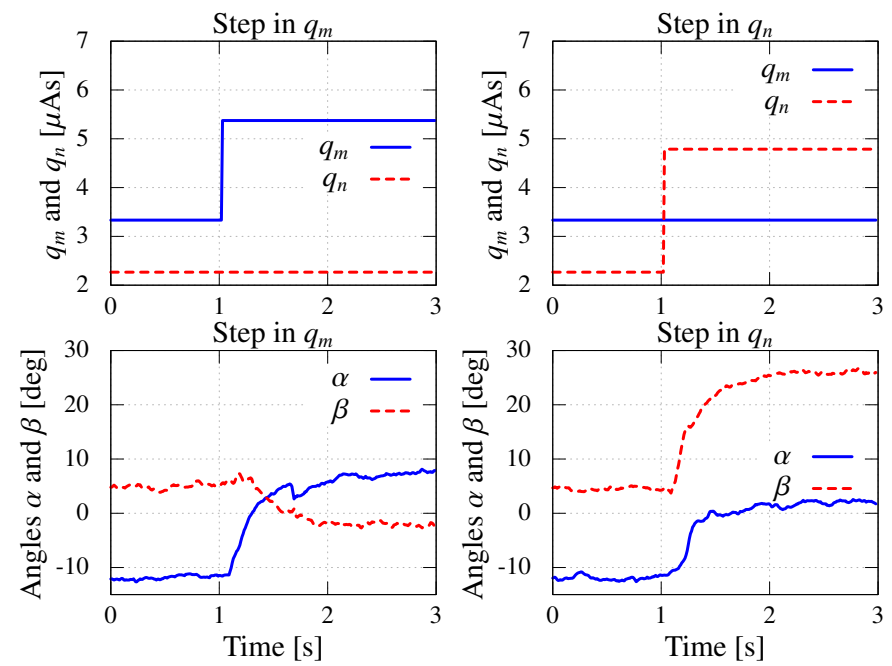

Fig. 6. Input step responses of the non-decoupled original system.
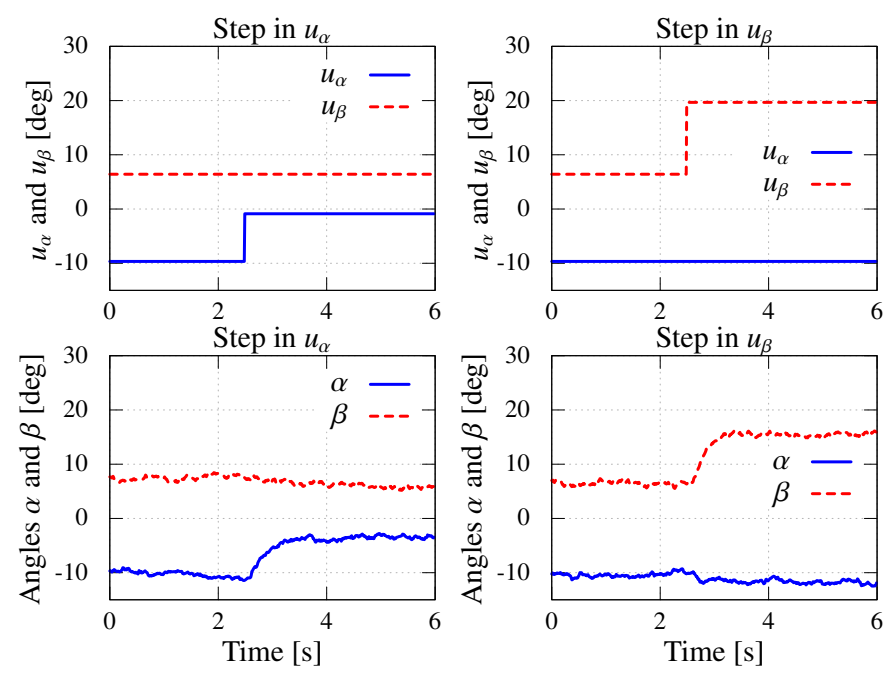

Fig. 7. Input step responses of the decoupled system.

decoupling. The accuracy of this model is questionable, especially for large dorsiflexion angles inside the rhomboid of Fig. 3. However, the reported input step responses of the open-loop decoupled system and the ILC results indicate the usefulness of this model for moderate dorsiflexion which is present during natural foot movements.

The assumption of a constant pass length $N$ will not hold for real walking. A first step towards an extension of the classical ILC theory for systems with variable pass length has already been developed in (Seel et al., 2011, 2013c) and can be used. An investigation of the proposed stimulation and control scheme with stroke patients walking on a treadmill is subject of our current research.

\section{REFERENCES}

Azevedo-Coste, C., Bijelic, G., Schwirtlich, L., and Popovic, D. (2007). Treating drop-foot in hemiplegics: the role of matrix electrode. In T. Jarm, P. Kramar, and A. Zupanic (eds.), 11th Mediterranean Conference on Medical and Biomedical Engineering and Computing 

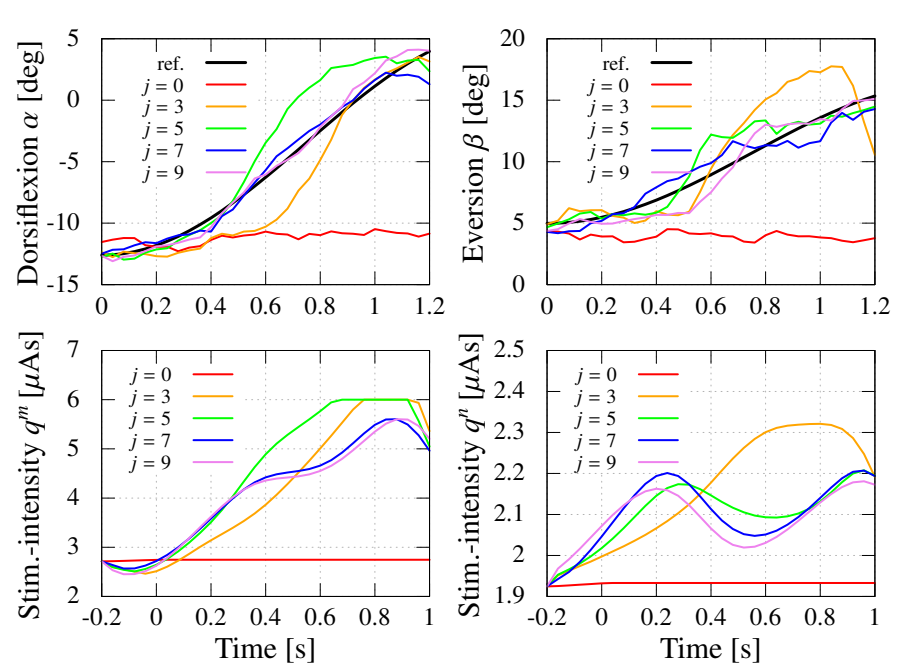

Fig. 8. ILC results for tracking desired dorsiflexion and eversion profiles.

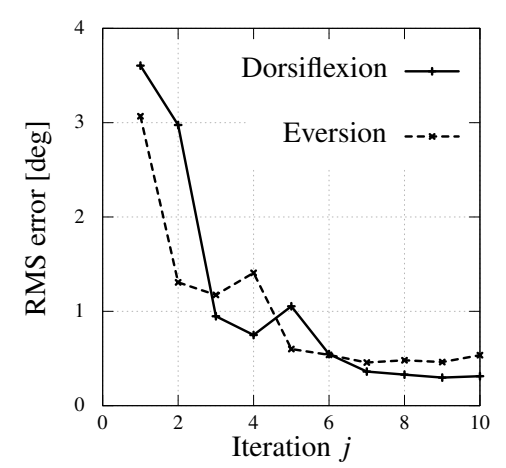

Fig. 9. Convergence of the RMS errors under ILC.

2007, volume 16, 654-657. Springer Berlin Heidelberg, Berlin, Heidelberg.

Bristol, E. (1966). On a new measure of interaction for multivariable process control. IEEE Transactions on Automatic Control, 11(1), 133-134.

Bristow, D., Tharayil, M., and Alleyne, A. (2006). A survey of iterative learning control. IEEE Control Systems Magazine, 26(3), 96-114.

Elsaify, A. (2005). A self-optimising portable FES system using an electrode array and movement sensors. Ph.D. dissertation, University of Leicester.

Hayashibe, M., Zhang, Q., and Azevedo-Coste, C. (2011). Dual predictive control of electrically stimulated muscle using biofeedback for drop foot correction. In Proc. of IEEE/RSJ International Conference on Intelligent Robots and Systems (IROS), 1731-1736.

Héliot, R., Mombaur, K., and Azevedo-Coste, C. (2013). Online CPG-Based gait monitoring and optimal control of the ankle joint for assisted walking in hemiplegic subjects. In Modeling, Simulation and Optimization of Bipedal Walking, 53-69. Springer.

Heller, B.W., Clarke, A.J., Good, T.R., Healey, T.J., Nair, S., Pratt, E.J., Reeves, M.L., van der Meulen, J.M., and Barker, A.T. (2012). Automated setup of functional electrical stimulation for drop foot using a novel 64 channel prototype stimulator and electrode array: Results from a gait-lab based study. Medical Engineering \& Physics, 35(1), 74-81.
Kotiadis, D., Hermens, H.J., and Veltink, P.H. (2010). Inertial gait phase detection for control of a drop foot stimulator: Inertial sensing for gait phase detection. Medical Engineering \& Physics, 32(4), 287-297.

Liberson, W., Holmquest, H., and Scott, M. (1961). Functional electrotherapy: Stimulation of the common peroneal nerve synchronised with the swing phase of gait of hemiplegic subjects. Arch Phys Med Rehabilitation, 42, 202-205.

Mourselas, N. and Granat, M.H. (2000). Correction of drop foot using a fuzzy logic controlled miniature stimulator. In Proc. of the 5th Annual Conference of the International Functional Electrical Stimulation Society. 140-141.

Nahrstaedt, H., Schauer, T., Shalaby, R., Hesse, S., and Raisch, J. (2008). Automatic control of a drop-foot stimulator based on angle measurement using bioimpedance. Artificial Organs, 32(8), 649-654.

Negard, N.O. (2009). Controlled FES-assisted gait training for hemiplegic stroke patients based on inertial sensors. Doctoral thesis, Technische Universität Berlin.

Reilly, J.P. and Diamant, A.M. (2011). Electrostimulation theory, applications, and computational model. Artech House, Boston.

Seel, T., Schäperkötter, S., Valtin, M., Werner, C., and Schauer, T. (2013a). Design and control of an adaptive peroneal stimulator with inertial sensor-based gait phase detection. In Proc. of the 18th Annual International FES Society Conference, 1-4. San Sebastian, Spain.

Seel, T., Schauer, T., and Raisch, J. (2011). Iterative learning control for variable pass length systems. In Proc. of the 18th IFAC World Congress, 4880-4885. Milan, Italy.

Seel, T., Schauer, T., and Raisch, J. (2013b). IMU-based joint angle measurement made practical. In Proc. of the Technically Assisted Rehabilitation - TAR Conference. Berlin, Germany.

Seel, T., Schauer, T., and Raisch, J. (2013c). Iterative Learning Control with Variable Pass Length applied to FES-Based Drop Foot Treatment (in German). at Automatisierungstechnik, 61(9), 630-37.

Sudlow, C.L.M. and Warlow, C.P. (1997). Comparable studies of the incidence of stroke and its pathological types results from an international collaboration. Stroke, 28(3), 491-499.

Valtin, M., Schauer, T., Behling, C., Daniel, M., and Weber, M. (2012). Combined stimulation and measurement system for array electrodes. In Biodevices 2012 International Conference on Biomedical Electronics and Devices, 345-349. Algarve, Portugal.

Wade, D.T., Wood, V.A., Heller, A., Maggs, J., and Langton Hewer, R. (1987). Walking after stroke. measurement and recovery over the first 3 months. Scandinavian Journal of Rehabilitation Medicine, 19(1), 25-30. 